\title{
THE PROJECT "PARSIFAL": PRANDTLPLANE ARCHITECTURE FOR THE SUSTAINABLE IMPROVEMENT OF FUTURE AIRPLANES
}

\author{
Vittorio Cipolla ${ }^{1, *}$, Aldo Frediani $^{1}$, Karim Abu Salem ${ }^{1}$, Marco Picchi Scardaoni ${ }^{1,2}$, \\ Vincenzo Binante ${ }^{3}$ \\ ${ }^{1}$ University of Pisa, Dept. of Civil and Industrial Engineering, Via G. Caruso 8, 56122, Pisa, Italy \\ ${ }^{2}$ Arts et Métiers ParisTech, Esplanade des Arts et Métiers, 33400 Talence, France \\ ${ }^{3}$ SkyBox Engineering S.r.1., , Via G. Caruso 8, 56122, Pisa, Italy
}

\begin{abstract}
The activities reported in this paper are part of the project "PARSIFAL" (Prandtlplane ARchitecture for the Sustainable Improvement of Future AirpLanes), funded by the European Community under the Horizon 2020 program and coordinated by the University of Pisa (Italy); the other partners of the project are: Delft University of Technology (The Netherlands), ONERA (France), Arts et Métiers ParisTech-ENSAM (France), DLR (Germany) and SkyBox Engineering (Italy). The paper presents a summary of preliminary design activities, carried out with the project PARSIFAL in order to study the introduction of box-wing aircraft, known as "PrandtlPlane", in the air transport system. PARSIFAL aims to investigate the adoption of the PrandtlPlane over short-to-medium routes, where aircraft compliant with the ICAO Aerodrome Reference Code "C" operate. According to such ICAO standard, the PrandtlPlane developed in PARSIFAL has a wingspan limited to $36 \mathrm{~m}$ but, if compared to reference aircraft such as B737 and A320 families aircraft, it can improve the passenger capacity from about 200 to more than 300 units. This paper presents the design steps performed for the definition of a "baseline configuration" of the PrP, introducing the requirements and describing both the conceptual and preliminary design phases, including the high fidelity investigations CFD and FEM analyses.
\end{abstract}

\section{Introduction}

According to several European studies ([1, 2, 3]), novel aircraft configurations as the ones shown in Fig. 1 represent one of the possible way to achieve the following:

- to fulfil the growth of air traffic demand, which is expected to double in next 20 years;

- to reduce $\mathrm{CO} 2$ and NOx emissions and noise per passenger-kilometres;

- to reduce to 4 hours the time required for a door-to-door journey within Europe.

The story of the box-wing configuration called "PrandtlPlane", or PrP (Fig. 1-right), has started in in 1924 when Ludwig Prandtl found that the so-called "best wing system" (BWS) has the property of minimizing the induced drag for given lift and wingspan ([4]). In 1990s, research performed at Pisa University have shown that a closed-form-solution of the minimum induced drag problem exists ([5]) and that it is possible to apply the Prandtl's concept to aircraft design $([6,7,8,9,10,11])$.

* Corresponding author: vittorio.cipolla@unipi.it 

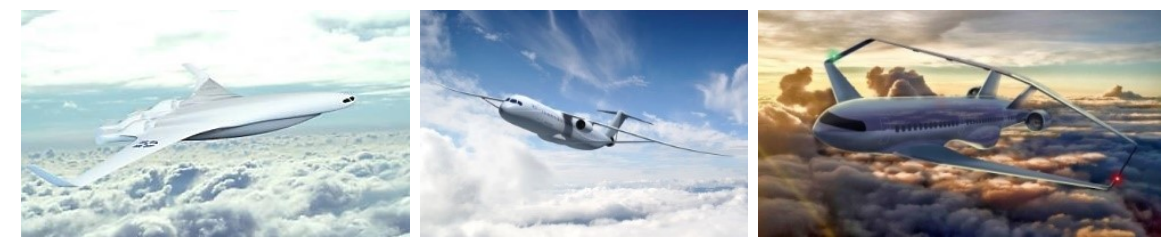

Fig. 1. Three examples of novel aircraft configurations. From left to right: the blended wing body (BWB), the truss-braved wings (TBW) and the box-wing or "PrandtlPlane" (PrP)

The present paper deal with the application of the PrP concept to commercial flight, in the framework of the EU funded project PARSIFAL ("Prandtlplane ARchitecture for the Sustainable Improvement of Future AirpLanes"), in which the future challenges of air transport are faced exploiting the aerodynamic advantages of the box-wing through the improvement of payload capabilities without increasing the overall dimensions of the aircraft.

\subsection{Top Level Aircraft Requirements}

The requirements adopted in PARSIFAL for the PrP design have been defined starting from the analysis of air traffic demand forecasts for 2030s, in which short-to-medium routes $(<4000 \mathrm{~km})$ will see the higher growth rate.

Today short-to-medium routes are flown mainly by single aisle aircraft with about 200 passengers, such as aircraft from Airbus 320 or Boeing 737 families. Therefore, in order to make use of the same airport infrastructures, the wingspan of the $\mathrm{PrP}$ has been limited to 36 $\mathrm{m}$, introducing the compliance with ICAO Aerodrome Reference Code "C" specification as a requirement. The choice of such aircraft category brings a set of constraints and typical mission parameters, which are summarized in the following Top-Level Aircraft Requirements (TLARs):

- number of passengers between 250 and 320 (high density),

- maximum range covered with maximum number of passengers of $2160 \mathrm{NM}$ (4000 $\mathrm{km})$,

- compliance with the ICAO Aerodrome Reference Code "4C" ("4" indicates a TakeOff Field Length $>1800 \mathrm{~m}$, whereas " $\mathrm{C}$ " refers to a wingspan $<36 \mathrm{~m}$ and wheel span between $6 \mathrm{~m}$ and $9 \mathrm{~m}$ ),

- initial cruise altitude of $36000 \mathrm{ft}$,

- Mach number in cruise flight greater than or equal to 0.78 .

\section{Conceptual design of the PrandtIPlane}

The conceptual design of the PrP has been performed considering the aforementioned TLARs, focusing the efforts the fuselage and the box-wing system.

As detailed in [12] and [13], the TLARs have been first used for the conceptual design of the fuselage, for which a single deck - double aisle layout with 8 seats abreast has been chosen. As a result, a $44 \mathrm{~m}$ long fuselage with a maximum payload capability of 308 seats in high density configuration and 12 LD3-45 containers have been defined (Fig. 2). 

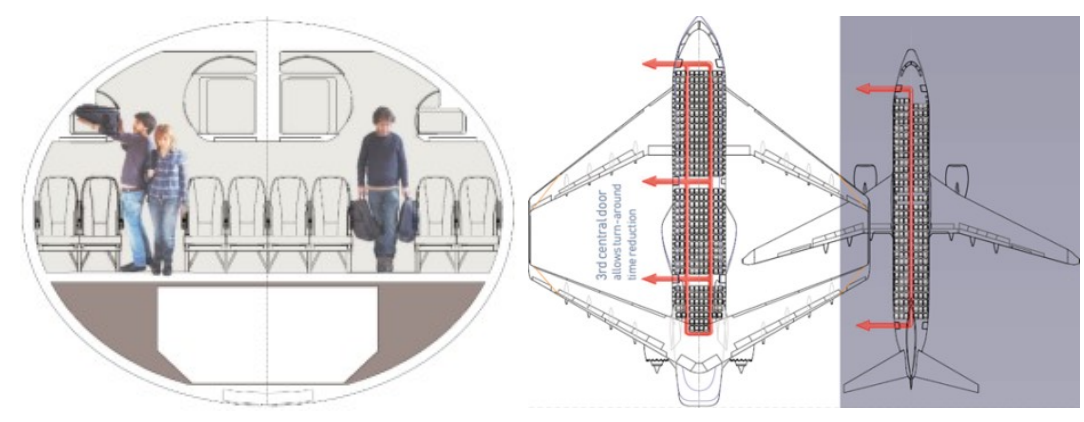

Fig. 2. Conceptual design of PrandtlPlane's fuselage and cabin

The conceptual design has been completed defining the box-wing design space, which has been explored by means of an optimization in-house developed tool, called AEROSTATE, which is based on the Vortex-Lattice Method (VLM) code AVL.

The optimization problem used for the PrP conceptual design is:

$$
\begin{gathered}
\min [-\mathrm{L} / \mathrm{D}(\mathbf{x})] \\
\mathrm{g}(\mathbf{x}) \geq 0 \\
\mathrm{~h}(\mathbf{x})=0 \\
\mathrm{lb}<\mathbf{x}<\mathrm{ub}
\end{gathered}
$$

where $\mathbf{x}$ is the vector of design parameters, each of them bounded in a specific interval $\left(\mathrm{l}_{\mathrm{b}}, \mathrm{u}_{\mathrm{b}}\right)$, the objective function is related to lift-to-drag ratio (L/D) in cruise condition, whereas $g(\mathbf{x})$ and $h(\mathbf{x})$ are constraint functions which take flight mechanics (e.g.: trim, longitudinal stability), aerodynamics (e.g.: lift distributions) and several geometric constraints into account.

At the end of the optimization process, AEROSTATE provide a set of feasible solutions white different values of L/D (Fig. 3).
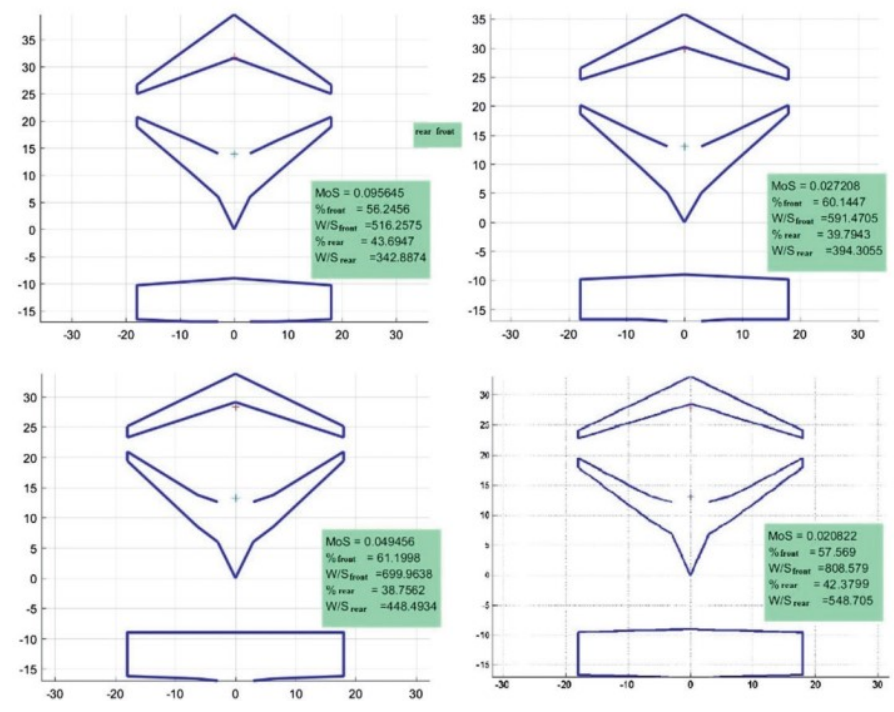

Fig. 3. Example of set of solutions provided by AEROSTATE 


\section{High fidelity investigations}

\subsection{Aerodynamics}

From the early stages of the project, it was necessary to investigate the aerodynamic behaviour of the PrP in cruise conditions with high-fidelity CFD tools. Such activity has been focused mainly on the effects of a set of design variables, such as the wing loading and geometric parameters, on transonic aerodynamics.

Test case configurations have been designed using AEROSTATE and Mach contours in cruise, as the ones shown in Fig. 4, have been investigated.

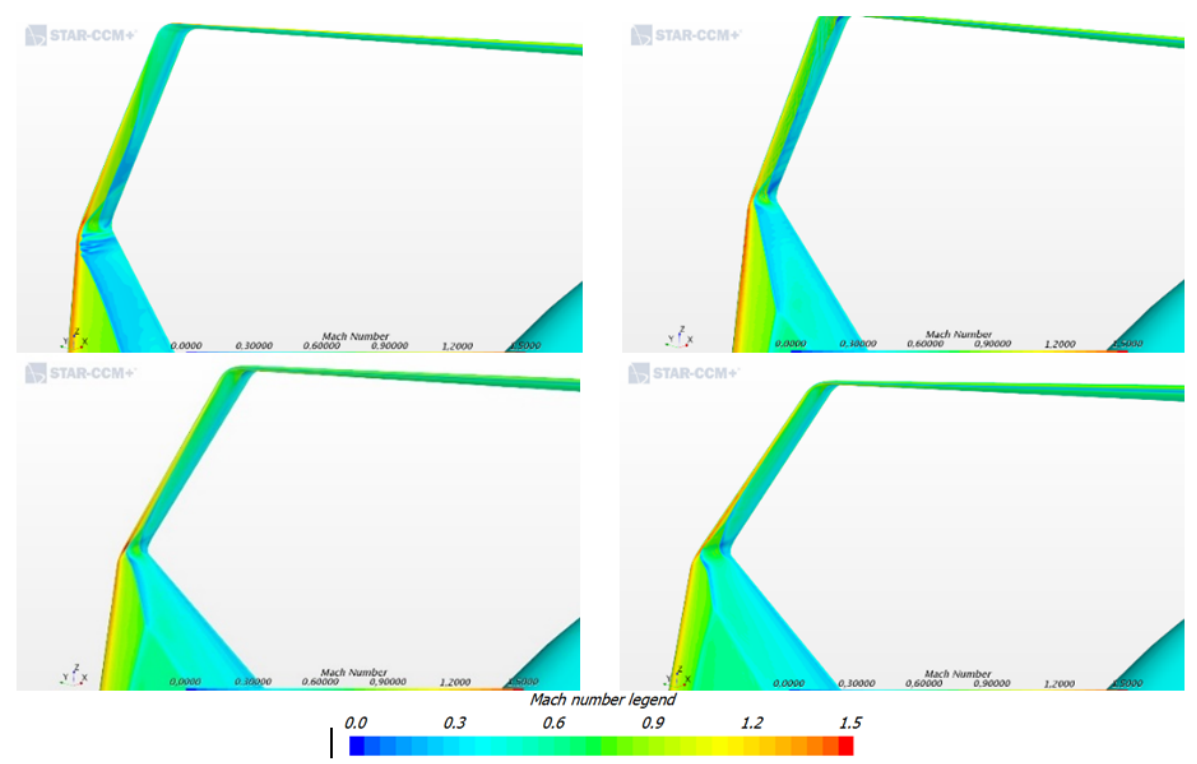

Fig. 4. Maps of local Mach number obtained through CFD simulations for different test case configurations

As described in [13], a trade-off between the performance in subsonic and transonic conditions is needed: in the first case, in fact, higher values of lift-to-drag ration are achievable only considering higher values of wing loading $([13,15])$, whereas in transonic flight, high values of wing loading can lead to drag rise conditions.

Information obtained from such CFD campaign has been used to properly calibrate the constraints and the boundaries of the AEROSTATE code, which is based on a potential aerodynamic solver, and so is not able to provide information on the compressibility effects on the drag in transonic flight.

Other investigations on geometric parameters' effects have been performed in order to improve the local aerodynamic performance, removing or limiting increases of drag due to the presence of shock waves.

\subsection{Structures}

Preliminary structural sizing has been performed with an in-house pre-processing tool, called WAGNER $([16,17])$, and commercial FEM software. Although it can be used for any aircraft configuration, WAGNER has been adopted in order to create detailed general FE model of PrP main structures, including stringers, frames, ribs, pressurization bulkheads, floor beams and floor struts (Fig. 5). 

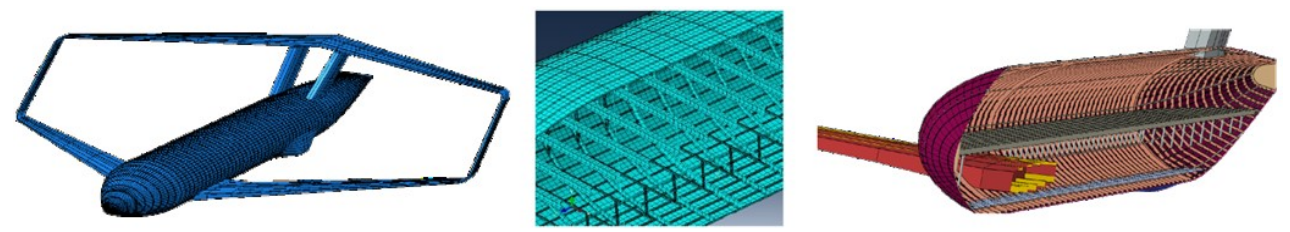

Fig. 5. Qualitative pictures of models generated by WAGNER

WAGNER has been used from the early stages of the project, in order to predict the total structural weight and CG position of the configuration, considering both aerodynamic and pressurization loads, under the main hypothesis of linear, homogeneous and isotropic elastic materials, which is suitable for metallic structures.

\subsection{Flight Mechanics}

Since there are different possible solutions to control and maneuver a PrP aircraft, in the initial phase of the project a reference layout has been selected: counter-rotating elevators are installed in the front and rear wing-root regions, ailerons are installed at the tip of each lifting surface, whereas high-lift devices are positioned in the reaming central region of each wing (Fig. 6).

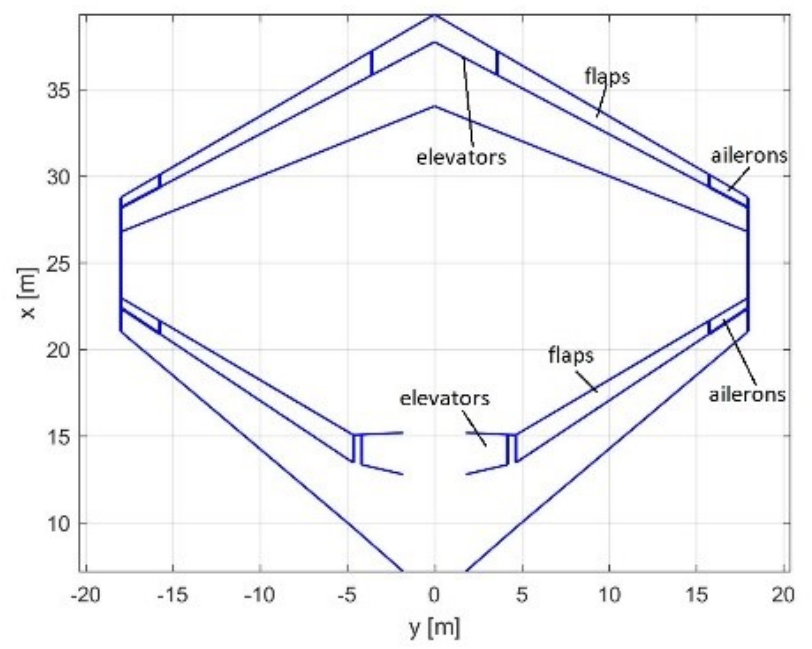

Fig. 6. Reference layout for PrP control surfaces

A VLM-based procedure for the preliminary sizing of control surfaces and high-lift devices has been defined for the PrP, considering the trim constraints in approach condition, as described in [13]. Such procedure results in an initial sizing of the control surfaces, and a preliminary estimation of the low speed performance of the aircraft, together with its aeromechanical characteristics of maneuverability and control.

Such information have been then used as input for higher fidelity assessments, in which CFD analyses have been performed to refine the evaluation of control derivatives. The pressure coefficient maps shown in Fig. 7 represent some of the obtained results, which have provided information useful to assess the behavior of this control systems layout, but also to validate or calibrate lower fidelity methodologies. 

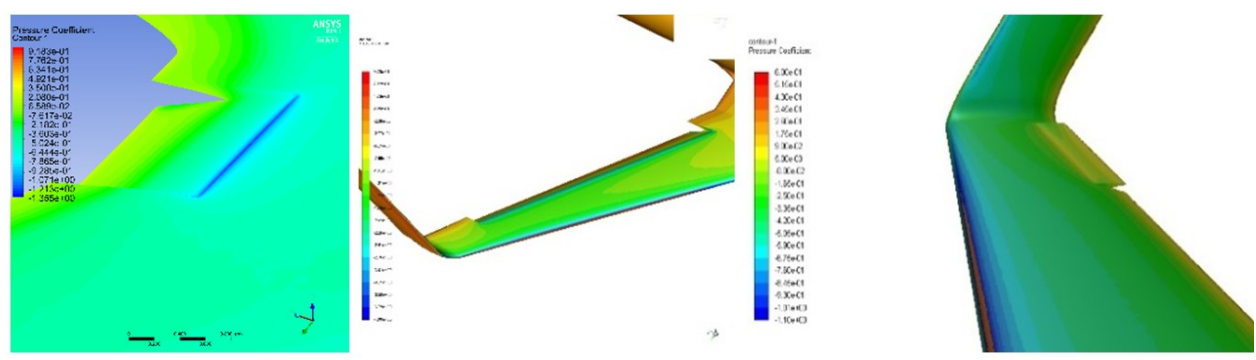

Fig. 7. Cp maps in approach condition for the PrP front wing with deflected elevator (left), flap (middle) and aileron (right)

\section{The Baseline PrandtIPlane configuration}

The preliminary design phase has started from the outcomes of the high fidelity investigations and, taking further aspects into account, such as landing gear design ([18]) and take-off performance analysis ([19]), has ended up in the definition of a baseline PrP configuration, which is briefly described in Table 1.

Table 1. Characteristics of the baseline PrP configuration

\begin{tabular}{|l|r|}
\hline Design conditions \\
\hline N. Passengers (high density) & 310 \\
\hline Flight Altitude [m] & 11000 \\
\hline Flight Speed [m/s] & 233 \\
\hline Cruise Mach & 0.79 \\
\hline Aircraft data & 267 \\
\hline Wing Area (front + rear) [m2] & 36 \\
\hline Wingspan (incl. winglets) [m] & 5.40 \\
\hline Fuselage Width [m] & 44 \\
\hline Fuselage Length [m] & 122 \\
\hline MTOW [tons] & 180 \\
\hline Take-off Thrust per engine [kN]
\end{tabular}

The baseline PrP has an important role in PARSIFAL, since it is not only the starting point for the advanced design loop, but it represent the reference configuration for assessing the impact assessment of the introduction of the PrP as a mean of transport.

Since one of the main drivers for the environmental and economic impact of an aircraft is the fuel consumption, the aerodynamic efficiency of the baseline PrP has been evaluated through CFD models ([20]). Considering a flight program with constant altitude $(\mathrm{h}=11000 \mathrm{~m})$ and Mach $(\mathrm{M}=0.79)$, the whole cruising phase has been simulated calculating:

- the fuel flow, i.e. the fuel burnt by each engine per unit time ;

- the thrust required to each engine;

- the total fuel burnt as the time-integral of fuel flow multiplied by the number of engines.

\section{Conclusions and further development}

The project PARSIFAL has come its final phase, in which the PrP designed to improve the payload capability of aircraft with wingspan below $36 \mathrm{~m}$, such as A320 and B737, 
while keeping the compatibility with present airport infrastructures, is under investigation from the standpoint of impact assessment.

The PrP considered is the one resulting from the $1^{\text {st }}$ design loop, which has started taking a set of Top Level Aircraft Requirements defined with a market-oriented approach into account. The paper, hence, has described both the conceptual design phase, in which the fuselage and the box-wing system have been object of low-fidelity studies, and the preliminary design.

Next steps of the research will be focused on the further development of the PrP configuration. The results of such activities will provide the input for the $2^{\text {nd }}$ design loop, which aims to provide an updated version of the PrP, which will be assessed in terms of environmental, logistic and economic impact.

\section{Acknowledgments}

The present paper concerns part of the activities carried out within the research project PARSIFAL ("Prandtlplane ARchitecture for the Sustainable Improvement of Future AirpLanes"), which has been funded by the European Union under the Horizon 2020 Research and Innovation Program (Grant Agreement n.723149).

\section{References}

1. European Commission. Flightpath 2050: Europe's Vision for Aviation. European Commission, Directorate General for Research and Innovation, Directorate General for Mobility and Transport (2011)

2. EREA. Study on the Air Transport System in 2050 from vision towards a planning for research and innovation. European Research Establishments in Aeronautics (2012)

3. ACARE, Strategic Research and Innovation Agenda (SRIA). Advisory Council for Aviation Research and Innovation in Europe (2017)

4. L. Prandtl, "Induced drag of multiplanes", NACA-TN-182 (1924)

5. A. Frediani, G. Montanari, "Best Wing System: An Exact Solution of the Prandtl's Problem", in "Variational Analysis And Aerospace Engineering", Springer Dordrecht Heidelberg London New York, pp 183-211. https://doi.org/10.1007/978-0-387-958576 11 (2009)

6. V. Cipolla, A. Frediani, E. Lonigro (2016). "Aerodynamic design of a light amphibious PrandtlPlane: wind tunnel tests and CFD validation". Aerotec. Missili Spaz. Vol. 94: 113. https://doi.org/10.1007/BF03404694 (2015)

7. A. Frediani, V. Cipolla, and F. Oliviero. "Design of a prototype of light amphibious PrandtlPlane", 56th AIAA/ASCE/AHS/ASC Structures, Structural Dynamics, and Materials Conference, AIAA SciTech Forum. https://doi.org/10.2514/6.2015-0700 (2015)

8. R. Cavallaro, L. Demasi, "Challenges, Ideas, and Innovations of Joined-Wing Configurations: A Concept from the Past, an Opportunity for the Future", Progress in Aerospace Sciences, Volume 87, Pages 1-93, ISSN 0376-0421, http://dx.doi.org/10.1016/j.paerosci.2016.07.002 (2016)

9. A. Frediani, V. Cipolla, E. Rizzo, "The PrandtlPlane Configuration: Overview on Possible Applications to Civil Aviation”. In: Buttazzo G., Frediani A. (eds) Variational 
Analysis and Aerospace Engineering: Mathematical Challenges for Aerospace Design. Springer Optimization and Its Applications, vol 66. Springer, Boston, MA. https://doi.org/10.1007/978-1-4614-2435-2_8 (2012)

10. A. Frediani, F. Oliviero, and E. Rizzo. "Design of an airfreight system based on an innovative PrandtlPlane aircraft", 56th AIAA/ASCE/AHS/ASC Structures, Structural Dynamics, and Materials Conference, AIAA SciTech Forum, https://doi.org/10.2514/6.2015-1186 (2015)

11. N. Beccasio, M. Tesconi, A. Frediani, "PrandtlPlane Propelled with Liquid Hydrogen: A Preliminary Study". In: Buttazzo G., Frediani A. (eds) Variational Analysis and Aerospace Engineering: Mathematical Challenges for Aerospace Design. Springer Optimization and Its Applications, vol 66. Springer, Boston, MA https://doi.org/10.1007/978-1-4614-2435-2_ 1 (2012)

12. V. Cipolla, A. Frediani, K. Abu Salem, M. Picchi Scardaoni, A. Nuti, V. Binante. "Conceptual design of a box wing aircraft for the air transport of the future". AIAA Aviation Forum. https://doi.org/10.2514/6.2018-3660 (2018)

13. A. Frediani, V. Cipolla, A. Abu Salem, V. Binante, M. Picchi Scardaoni, "Conceptual design of PrandtlPlane civil transport aircraft". Proceedings of the Institution of Mechanical Engineers, Part G: Journal of Aerospace Engineering. https://doi.org/10.1177/0954410019826435 (2019)

14. V. Cipolla, A. Frediani, K. Abu Salem, V. Binante, E. Rizzo, M. Maganzi, "Preliminary transonic CFD analyses of a PrandtlPlane transport aircraft". Transportation Research Procedia, 29: 82-91. https://doi.org/10.1016/j.trpro.2018.02.008 (2018)

15. K. Abu Salem, V. Cipolla, M. Carini, M. Méheut, S. Kanellopoulos, V. Binante and M. Maganzi "Aerodynamic design and preliminary optimization of a commercial PrandtlPlane aircraft", Proceedings of $8^{\text {th }}$ EUCASS Conference, Madrid (2019)

16. M. Picchi Scardaoni, V. Binante, V. Cipolla, "WAGNER: a new code for parametrical structural study of fuselages of civil transport aircraft". Aerotec. Missili Spaz. 96: 136. https://doi.org/10.1007/BF03404748 (2017)

17. M. Picchi Scardaoni, V. Cipolla, V. Binante, Overall Preliminary Sizing and Optimisation of the Metallic Structures of a PrandtlPlane Civil Transport Aircraft, Proceedings of $8^{\text {th }}$ EUCASS Conference, Madrid (2019)

18. A. Nuti, F. Bertini, V. Cipolla, G. Di Rito, "Design of a Fuselage-Mounted Main Landing Gear of a Medium-Size Civil Transport Aircraft", Aerotec. Missili Spaz. Vol. 97: 85. https://doi.org/10.1007/BF03405803 (2018)

19. K. Abu Salem, G. Palaia, M. Bianchi, D. Zanetti, V. Cipolla, V. Binante, "Preliminary take-off analysis and simulation for a PrandtlPlane commercial aircraft", Proceedings of the XXV AIDAA International Conference, Rome (2019)

20. M. Carini, M. Méheut,, "CFD analysis of a box-wing architecture within the framework of the PARSIFAL project", ODAS (2018) 